\title{
La Administración del Nuevo Laborismo: sumando y actuando conjuntamente
}

\section{R. A. W. Rhodes}

En los últimos veinte años la reforma de la Administración pública ha sido continua. En el artículo «Comprendiendo la gobernabilidad (governance)» expongo un relato de la reforma del sector público en el Reino Unido bajo el gobierno conservador. Este artículo se centra en los principales desarrollos que se han producido en el gobierno central desde que el nuevo laborismo ocupó el poder en mayo de 1997 bajo la figura de Tony Blair ${ }^{1}$.

El Nuevo Laborismo fue lento a la hora de ponerse a modernizar la Administración Pública. En un principio se anunció un «Libro Blanco» («White Paper») sobre Una mejor gobernabilidad, para el otoño de 1997. Limpiaría la política; expulsaría la basura y la ineficiencia; mejoraría la regulación; prestaría atención a la gente en desarrollo de los servicios; proporcionaría una mayor transparencia y responsabilidad; utilizaría el potencial de IT para proveer los servicios, no sólo la información; y tiraría abajo las barreras institucionales para trabajar conjuntamente. El Canciller del Ducado de Lancaster prometió una iniciativa radical que pondría en marcha la visión del aspecto del gobierno dentro de quince años. Este proyecto se retrasó repetidamente desde principios de 1998 a junio o julio. Pecó de no decirlo todo cuando partió de que la reforma del sector público no era una prioridad.

En el «Libro Blanco» («White Paper») que finalmente apareció en marzo de 1999 bajo el título Modernizando el Gobierno, el gobierno anunció tres objetivos:

- asegurar que la realización de políticas fuera más conjunta y estratégica;

- asegurar que los usuarios de los servicios públicos iban a ser una prioridad.

- proveer servicios públicos de alta calidad y eficientes.
Su programa de reformas tiene cinco compromisos claves:

- realización de políticas: mirar hacia adelante en el desarrollo de políticas para producir resultados importantes, y no actuar solamente ante presiones a corto plazo;

- una Administración pública que responde; para proporcionar servicios públicos que se enfrenten a las necesidades de los ciudadanos, y no a la conveniencia de los proveedores de servicios; - servicios públicos de calidad: para proporcionar servicios públicos eficientes y de alta calidad y no tolerar la mediocridad;

- gobierno acorde con la era de la información: utilizar las nuevas tecnologías para hacer frente a las necesidades de los ciudadanos, y de las empresas, y no quedarse detrás en los desarrollos tecnológicos;

- el Servicio Público: valorar la Función Pública, y no denigrarla (Cm 4310, 1999: 6 y 13) ${ }^{2}$.

La sencilla evaluación que cabe hacer de estos compromisos es que la política del gobierno era pragmática y obtuvimos más de lo mismo. De hecho, el estilo «con cuentagotas» de reforma ocultó una cambio pasando de poner especial énfasis en los mercados a ponerlo en las redes. Más abajo me detengo algo más en este argumento después de describir brevemente tanto los cambios alcanzados hasta el momento como los cambios que se ha previsto realizar y que se establecen en el «White Paper».

\section{Variedades de la reforma}

La reforma ha adoptado multitud de formas durante las dos últimas decadas, y lo escrito al respecto sugiere que tiene 
seis partes: privatización, marketización, administración corporativa, regulación, descentralización y control político. Definiré estos términos a medida que analizo los cambios, centrándome en los efectos de la Administración Pública, en particular, más que en el sector público en terminos generales.

La privatización se refiere a la venta de activos al sector privado. En cuanto a si la política resultó ser un éxito -y uno puede argumentar sobre estos objetivo varios y la medida en que éstos se alcanzan - y no puede haber ningún tipo de duda sobre la escala del programa. El gobierno vendió más de 50 empresas principales, obteniendo unos 64 billones de libras esterlinas para compensar el recorte de impuestos. El grado de privatización necesariamente se reducirá bajo el Nuevo Laborismo, simplemente porque ya se han vendido muchas empresas públicas. Sin embargo, no queda descartado, ideológicamente - por ejemplo, el Nuevo Laborismo vendió el 60\% de sus activos en la «Commonwealth Development Corporations».

La privatización tiene un resultado evidente para la función pública: las cifras se redujeron de forma importante, de un máximo de 762.000 en 1976 a 481.000 en 1998 (una bajada del $38 \%$ ). De forma muy significativa, los funcionarios no industriales bajaron de 571.000 a 430.000 (una bajada del 25\%) mientras que los funcionarios industriales bajaron de 209.000 a 33.000 (una bajada del 84\%), que supone un 63\% de la bajada global de las cifras de los empleados públicos.

La marketización hace referencia al uso de mecanismos de mercado para proveer los servicios públicos. En el Reino Unido, el término cubre principalmente contracting-out (por ejemplo, la realización de propuestas competitivas de forma obligatoria en el gobierno local); quasi-mercados bajo la apariencia de la partición entre comprador-proveedor (por ejemplo, en el Servicio Sanitario Nacional); y experimenta con fórmulas de bonos (por ejemplo, en la educación infantil). Bajo el Nuevo Laborismo, la marketización se reducirá. La división entre comprador-proveedor en el ámbito de la salud y de los bonos de guarderías se abolirá. Sin embargo, el retraso (mora) propuesta en el contracting-out (Hansard, 22 de mayo de 1996, col. 311) no tuvo lugar. Por ejemplo, la iniciativa de financiación privada (PFI) en materia de salud supone la construcción por parte del sector privado de hospitales y arrendarlos de nuevo al sector público por un período de hasta sesenta años. Unos 31 hospitales también se financiarán. En breve, la política es que la marketización «tiene un papel que desempeñar, de nuevo no por dogmatismo pero por pragmatismo, porque queremos la mejor utilización del dinero (value for money) (Cm 4310, 1999: $35)$.

La Gestión Empresarial se refiere a la introducción de aproximaciones propias del sector privado en el ámbito del sector público. Se centra en bands-on, administración profesional; obje- tivos explícitos, estándares y medidas del rendimiento; gestión por resultados; el valor por el dinero; y, más recientemente, la cercanía al consumidor. Existen muchas variaciones en esta lista, y la mayor parte de ellas tienen su origen en una preferencia por los estilos de administración del sector privado, poniendo el énfasis en la medición de la actuación en general y en la medida del output en particular. Por ejemplo, los funcionarios públicos ahora tienen contratos y construyen sus propios currículums más que otros dirijan sus carreras por ellos (ver, por ejemplo, Cm 2627, 1994; Cm 2748, 1995).

La administración corporativa tiene una larga historia en el Reino Unido. Su signo de referencia en la actualidad lo constituyen las «tres e», de economía, eficiencia y efectividad, con la economía siempre presente. Este primer énfasis cambió para hacer los servicios transparentes para sus consumidores a través de mecanismos tales como la Carta de Derechos de los Ciudadanos. La política del Nuevo Laborismo es una política pragmática, con un gobierno que apoya iniciativas tales como Benchmarking que tiene como objetivo medir y mejorar la calidad; e «Inversores en gente», que marcan estándares para la gestión de recursos humanos (Cm 4310, 1999: 39). Ambos continúan con la mini-tradición de importar prácticas de gestión del sector privado al sector público.

El Nuevo Laborismo ha reinventado la Carta de Derechos de los ciudadanos como un «Primer Servicio». Por ejemplo, el gobierno ha establecido un Panel de personas que comprende 5.000 individuos de todo tipo para que expongan sus puntos de vista acerca de la mejora de la provisión de servicios (Cabinet Office, 1998; $\mathrm{Cm}$ 4310, 1999: 25 y 29). También tiene una conmovedora fe en la capacidad de la información tecnológica para mejorar la calidad y responsabilidad de la provisión de servicios a nivel local (Cm 4310, 1999: ch. 5). El informe de Sir Richard Wilson dirigido al Primer Ministro sobre la reforma de la Administración Pública (Cabinet Office, 1999c) muestra claramente la importancia que el Nuevo Laborismo da a que haya altos niveles de actuación y de valor del dinero por parte de la Administración Pública. El gerencialismo avanza hacia el nuevo milenio.

$\mathrm{Al}$ redibujar las fronteras del Estado en los años ochenta, el gobierno británico trató de endurecer su capacidad para regular y controlar a las instituciones, sus políticas y la implementación de sus políticas. El gobierno sustituyó la regulación por la propiedad, y al hacerlo multiplicó a los vigilantes de los nuevos monopolios del sector privado. La «explosión controladora» se refiere a las formas de regulación interna y externa. Abarca la gestión y el control y la evaluación financiera, con sistemas relacionados que aseguran la calidad y «una mentalidad diferenciada de control de la Administración», que deja de lado la confianza y se centra en cuantificar formas expertas de control externo y ex-post ${ }^{3}$. 
El Nuevo Laborismo no ha legislado para cambiar el sistema de regular las utilidades públicas. Margaret Becket, cuando fue Secretaria de Estado en el Departamento de Comercio e Industria, anunció que su departamento llevaría a cabo un informe interdepartamental de la regulación de las utilidades; pero no provocó cambios significativos. Los reguladores del gas y de la electricidad se han fusionado, al igual que lo han hecho los reguladores del ferrocarril, para crear una Nueva Autoridad Estratégica del Ferrocarril. Las declaraciones públicas ponen de relieve la responsabilidad frente a los consumidores y critican los incrementos de salario fatcat. El Nuevo Laborismo ha establecido una Unidad de Mejor Regulación en el «Cabinet Office» y a un equipo técnico para mejorar «la efectividad y credibilidad de la regulación asegurando que sea necesaria, justa, y que se la puedan permitir, además de sencilla de entender y administrar, tomando en consideración las necesidades de las pequeñas empresas y de la gente normal». Resumiendo, ha puesto especial énfasis en la reducción de la burocracia, más que en, por ejemplo, la efectividad a la hora de proteger los intereses de los consumidores.

$\mathrm{El}$ aumento de la regulación interna es masivo. Christopher HooD y sus colegas identifican 134 organismos que gastan 776 millones de libras esterlinas al año en costes de rodaje y, más o menos, gastando lo mismo en costes de consentimiento. Los ejemplos de tales organismos van desde la Oficina de Auditoría Nacional y la Inspección de las Prisiones, al Comisionado Parlamentario para la Administración, La Oficina de Servicio Público y el Consejo para Fondos de la Educación Superior. Si ha ocurrido algo, se ha acelerado el crecimiento bajo el Nuevo Laborismo. Hood identifica al menos doce principales nuevas organizaciones que se han creado a partir de marzo de 1999. Éstas incluyen la Inspección de Beneficio del Fraude (86 personas y 3,4 millones de libras esterlinas); el régimen del «Mejor Valor» para el gobierno local, que incluye dos inspecciones; y la Comisión para la Mejora de la Salud y el Instituto Nacional de Excelencia Clínica para el NHS. El coste de los cambios del Nuevo Laborismo se estima en, al menos, 20 millones de libras esterlinas al año.

HooD también detecta un cambio significante en la filosofía reguladora bajo el Nuevo Laborismo (ver Cm 4310, 1999: 37-9). Hay una creciente consciencia de los costes de la regulación y de la necesidad de consistencia de la práctica entre reguladores. Por ejemplo, el gobierno ha establecido el Forum de la Auditoría Pública para compartir las ideas respecto de las buenas prácticas ( $\mathrm{Cm} 4310,1999$ : 37). Más significativo aún es la tendencia a «dar vigor a la autorregulación»; es decir, una regulación más formal y externa para los malos actores, pero lo que Tony Blair denomina «regímenes de inspección de leve control» para buenos actores. Un buen ejemplo es el régimen de «Mejor Valor» para el gobierno local, bajo el que la mayor parte de las autoridades locales corregirán sus propios fallos, pero unos pocos malos actores se someterán a una fuerte intervención central ${ }^{4}$.

El estricto clima financiero fue testigo de cómo la Tesorería se volvió más poderosa. El gobierno conservador introdujo varios cambios en el presupuesto y en la administración financiera para endurecer su control sobre el gasto total, a la vez que aumentó de forma ostensible la delegación financiera. Sin embargo, la discreción financiera fue meramente una ilusión. La cruda realidad de todos los días fue el control financiero, que yacía en el centro del paquete de reforma del Reino Unido y que continúa habiéndolo bajo el Nuevo Laborismo $(\mathrm{Cm} 4310$, 1999: 36-7). Los planes de gasto público se fijan para un período de tres años. La contabilidad de los recursos busca unir los recursos a los outputs políticos. El Informe de Gasto Integrado identifica las prioridades del gobierno a la hora del gasto ( $\mathrm{Cm}$ 4011, 1998). La estrategia consiste en tener objetivos y otras medidas para asegurar que esas prioridades se traducen efectivamente en servicios. Así, los patrones de gasto se cambiaron y los «Acuerdos de la Administración Pública» (Cm 4181, 1998; Cm 4310, 1999: 36-7) se introdujeron para imponer la disciplina necesaria.

La descentralización comprende tanto la desconcentración como el traspaso de competencias. La desconcentración hace referencia a la redistribución de las responsabilidades administrativas en el gobierno central. Por su parte, el traspaso hace referencia al ejercicio de autoridad política por instituciones establecidas y elegidas en el ámbito de áreas definidas por características de la comunidad. En el Reino Unido, la mayor parte de las reformas de los años ochenta y noventa buscaban desconcentrar la autoridad administrativa, por ejemplo, en favor de las agencias. El traspaso fue un aspecto de la reforma del sector público en otros lugares, por ejemplo, en Dinamarca, Francia y España. Con el advenimiento del gobierno laborista, el traspaso se convirtió en una prioridaad en Gran Bretaña.

La agencificación supone crear agencias semiautónomas responsables de la administración operacional. La noción clave aquí es la de separar la administración operacional del centro de realización de políticas o departamento central, de tal forma que la agencia dispone de libertad para administrar. Ésta constituye una clásica doctrina de la Administración Pública. Para enero de 1998 el programa estaba ya casi completado, con 138 agencias que empleaban a un $77 \%$ del funcionariado civil $(\mathrm{Cm}$ $3889,1998)$.

Después de iniciales dudas, el Nuevo Laborismo aceptó estas agencias; la única cuestión pendiente es la de si resistirán la interferencia en las mismas que se está produciendo. Ciertamente, parece probable que sucumban ante la tentación, tal y como lo hicieran sus predecesoras. Así, Jack Straw, como Secretario Nacional, asumió que la responsabilidad de hacer frente a estas preguntas recayera sobre el Servicio de Prisión 
en vez de dejar esto al Director General de las agencias. No hay consenso respecto del grado de desconcentración de las agencias. DAY y KLEIN concluyeron que en el NHS, la política ha emigrado del departamento central a la agencia ${ }^{5}$. Para los jefes ejecutivos de muchas agencias, la realidad de todos los días es una interferencia constante por el departamento jefe, Oficina de Tesorería y «Cabinet Office». Tal y como señaló un jefe ejecutivo: «Soy un funcionario civil y no puedo decirlo» (Cabinet Office, 1994: 31). Ahora existen multitud de agencias, pero la habilidad ministerial para dejarla en paz sigue siendo como siempre variable. La descentralización sigue siendo evasiva.

Acerca del traspaso, los funcionarios civiles escoceses fueron trasladados al Parlamento de Edinburgo, pero, como parte del personal de la Ejecutiva escocesa, siguen siendo parte de una Administración pública unificada. Hay tensiones evidentes en tales tipos de lealtades duales. El gobierno reconoce que existe un problema, asegurando que «la lealtad en último término de los funcionarios civiles será hacia la Corona», pero, «en la práctica la lealtad de los funcionarios civiles individuales será hacia cualquier administración a la que estén sirviendo» y «seguirán recibiendo órdenes de sus ministros de departamento» (División de personal, Cabinet Office, «Traspaso y la Administración Pública», nota 2, 1998). Parece seguro que el funcionariado civil del Reino Unido estará cada vez menos unificado.

Escocia continúa recibiendo su parte de gasto público y tiene el poder para variar el impuesto sobre la renta en 3 peniques por cada libra esterlina. El Parlamento escocés fija la estructura general para otros cuerpos públicos escoceses, incluyendo el gobierno local electo. Escocia se ve ahora imbuida en un cambiado y cambiante marco de lazos institucionales. Una Comisión Ministerial Conjunta (JMC) formada por ministros del gobierno del Reino Unido, ministros escoceses, miembros de la Cámara de la Asamblea galesa y ministros en la Comisión Ejecutiva de Irlanda del Norte, permitirá una coordinación centralizada (Cm 4444, 1999: 9-11). Tendrá en consideración asuntos no desarrollados que chocan con las responsabilidades traspasadas y viceversa. En asuntos de la Unión Europea, la JMC coordinará las administraciones traspasadas y que pueden tener oficinas en Bruselas, pero que «apoyará y hará avanzar únicamente la línea de negociación del Reino Unido» (Cm 4444, 1999: 20-3). Sigue sin estar claro cuál es el papel del funcionariado civil y las formas en que la responsabilidad ministerial funcionará.

Antes de 1972, el gobierno local podía describirse como parte de la Constitución. No se podría hacer tal tipo de consideración hoy. El gobierno británico central ha ejercitado el mayor control existente sobre las autoridades locales, especialmente respecto de su gasto. La política era centralizar, incluso si los resultados no eran siempre como se esperaba que fueran. Desde que se produjeron numerosos engaños, el nuevo gobier- no laborista decidió que no habría asambleas regionales en Inglaterra. También este nuevo gobierno está nervioso respecto a traspasar poder al gobierno local, porque está decidido a mantener el control respecto del gasto público. La pieza central del «White Paper» del gobierno respecto del gobierno local (Cm 4014, 1998) son los alcaldes electos, y su tema es la renovación de la democracia; pero pone de manifiesto una evidente resistencia a dotar de poder a las autoridades locales.

No es habitual que un artículo acerca del funcionariado civil hable del gobierno local, pero es un aspecto del «White Paper» (ver, por ejemplo, Cm 4310, 1999: 11-12), porque la llamada a favor de un conjunto es un aspecto distintivo en las reformas. Tony Blair expuso sucintamente las metas — «los problemas conjuntos necesitan actuaciones conjuntas»- $-\mathrm{y}$ este tema recorre el «White Paper» de «Modernizando el Gobierno» con su frecuentes referencias a gobierno «conjunto» y a bolistic governance $(\mathrm{Cm} \mathrm{4310,} \mathrm{1999:} \mathrm{6,} \mathrm{7,} \mathrm{10-11,} \mathrm{15,} \mathrm{16,} \mathrm{20,} \mathrm{23,} \mathrm{24,}$ $27,32,33,40,45,46,53$ y 56 ). De tal forma que los servicios deben ser efectivos y estar coordinados, y los principios del gobierno conjunto también se aplican a las organizaciones voluntarias y a organizaciones del sector privado. La «Cabinet Office» lleva esta idea incluso más allá fijando estándares para la realización de políticas «modernizadas» que incluyen el caracter conjunto o integrado (Cabinet Office, 1999b: ch. 9 y Anexos A y B). Defiende que los decisores políticos todavía están «empezando a manejarse».

El carácter conjunto puede adoptar varias formas. Por ejemplo, hay programas basados en áreas o «zonas de acción» (27 en salud, 25 en educación) uniendo el gobierno central y local, las autoridades sanitarias, y las organizaciones voluntarias y del sector privado; y los programas piloto, centrados en grupos tales como «Un Mejor Gobierno para la gente mayor» piloto $(\mathrm{Cm}$ 4310, 1999: 18, 26-7 y 29). El Estado es un socio capaz, que une y mueve las redes flexibles, y el funcionariado civil debe adaptarse. Ya los jargon breeds —diplomáticos, papeles para ampliar las fronteras, reticulistas-, pero cualquiera que sea la etiqueta que se les ponga, la tarea es la de construir puentes entre las organizaciones implicadas diseñando políticas y proveyendo servicios.

Los esfuerzos de los ministros por reasegurar el control político sobre el funcionariado civil son un aspecto común de la reciente reforma del sector público en los sistemas de Westminster. Con anterioridad a las elecciones generales de 1997, existía la preocupación de que el funcionariado civil actuará demasiado tiempo bajo el mando de un único partido político, pero la transición hacia el nuevo laborismo se produjo sin ningún problema - sólo hubo una jubilación-y el Primer Ministro escribió al entonces Director del «Home Civil Service», Sir Robin Butler, dándole la enhorabuena respecto de la entrega del poder. No ha habido ninguna noche de los cuchillos largos para los secretarios permanentes. El nuevo encargado del Fun- 
cionariado Civil, Sir Richard Wilson, es de forma reconocida un mandarín. Sin embargo, varios secretarios permanentes se retirarán en los próximos años, de tal forma que el Primer Ministro tendrá amplias posibilidades para efectuar cambios.

Se produjo un aumento importante de los consejeros políticos, especialmente en la Unidad de Política Número Diez, que ahora no incluye funcionarios. El objetivo era alcanzar una mejor coordinación de la política del gobierno. Sir Richard Wilson dirigió un informe a la «Cabinet Office» que pretendía convertirla en el centro corporativo del funcionariado civil a la vez que endurecer la coordinación interdepartamental. Las innovaciones específicas incluyen la fusión de la Oficina de Servicio Público con el resto de la Cabinet Office, estableciendo una Unidad de Innovación y Actuación para mejorar la coordinación de la entrega de servicios, e incorporando el Colegio de Servicio Público en un Nuevo Centro de Administración y de Estudios Políticos que conjuntamente adiestrará a los ministros y funcionarios y dirigirá la implementación del «White Paper» (Cm 4310, 1999: 20, 57).

Estos cambios deberían interpretarse como centralización porque el tema central en ellos es la mejora de la administración corporativa del funcionariado civil ( $\mathrm{Cm} \mathrm{4310,} \mathrm{1999:} \mathrm{20).} \mathrm{Tal}$ y como argumenta Peter Hennessy, la Unidad Número Diez y la Oficina del Gabinete dirigen la realización de políticas del gobierno, inicialmente con Peter Mandelson y ahora con Mo Mowlam como «ejecutor del gabinete». El apoyo a este centro proactivo se da por parte de 150 task forces, grupos de consulta e informes diseñados para hacer frente, por ejemplo, a los «asuntos complicados» (wicked issues), que van más allá de las fronteras ministeriales. Así, «el número es una unidad omnipresente en informes serios sobre política». El ejemplo por excelencia es el Código Ministerial (Oficina del Gabinete 1997) y sus órdenes que exigen que todos los informes principales, publicaciones de prensa y declaraciones políticas deberían aclararse con el Número $10^{6}$.

El «White Paper» Modernizando al Gobierno llega a la conclusión de que el funcionariado civil hace frente a siete retos:

- Implementar la reforma constitucional de tal forma que preserve un funcionariado civil unificado y asegure un trabajo cercano y coordinado entre el gobierno del Reino Unido y las administraciones traspasadas.

- Conseguir personal en todos los departamentos para integrar la dimensión de la Unión Europea en el pensamiento político y las decisiones de esta índole.

- Centrando el trabajo en los servicios públicos para mejorar así su calidad, hacerlos más innovadores y responsables frente a los usuarios y asegurar que estos servicios son prestados de forma eficiente y conjunta.

- Crear una cultura en el marco del funcionariado civil que sea más innovadora y menos adversa al riesgo.
- Mejorar el trabajo en colaboración superando las barreras organizacionales.

- Administrar el funcionariado civil, de tal forma que se esté equipado para hacer frente a estos retos.

- Pensar hacia adelante estratégicamente, hacia futuras prioridades» (Cm 4310, 1999: 56).

Existe un Plan de Acción para implementar las propuestas del «White Paper» y el gobierno publicará periódicamente lo diversos «hitos» a medida que se van alcanzando esos retos (Oficina del Gabinete, 1999a).

Necesitamos dar respuesta a dos preguntas: ¿Consiguen estas reformas vencer las consecuencias no intencionadas de las reformas previas? ¿Permitirán al gobierno estas reformas alcanzar sus objetivos fijados?

\section{Consecuencias no intencionadas}

Muchos de los problemas surgieron de la reformas del sector público llevadas a cabo por el gobierno conservador, principalmente: la fragmentación, el steering, la responsabilidad, la politización y los roles administrativos.

El resultado más evidente del nuevo sistema ha sido la fragmentación institucional. Típicamente, los servicios se prestan ahora a través de una combinación del gobierno local, organismos con objetivos determinados, el sector voluntario y el sector privado. Existen en la actualidad 5.521 organismos de objetivo determinado que gastan al menos 39 billones de libras esterlinas al año, y respecto de los cuales los ministros realizan 70.000 patronage appointments. iEste sector es incluso mayor que el propio gobierno local! El «White Paper» Modernizando al Gobierno, proporciona su propia ilustración del gran número de organizaciones que están implicadas en proveer cuidado domiciliario a largo plazo (Cm 4310, 1999:24). Por tanto, como no había sucedido nunca antes, ahora, la prestación del servicio depende de la conexión entre organizaciones, y el interés central del «White Paper» en un gobierno integrado supone una respuesta directa a este problema.

JENKINS argumenta que, junto con los otros cambios, el gobierno no fortaleció la capacidad estratégica. Como consecuencia de ello, las agencias trabajan en un «vacío político» y el steering ocurre «a través de un sistema de gestión de la crisis y evitación de la culpas». Sir Robin ButLer puso de manifiesto estas mismas preocupaciones respecto de la realización de políticas a nivel central: «Es esencial que no se alcance el punto en que los departamentos individuales y sus agencias 
se conviertan en simples elementos desconectados en el marco general del sector público, sin... verdaderos mecanismos de trabajo para la coordinación de políticas» (énfasis añadido) ${ }^{7}$.

La anterior Dirección Sanitaria Regional de Yorkshire proporciona un buen ejemplo de los límites de la habilidad del centro para virar (steer). Cuando el Comité parlamentario de Cuentas Públicas investigó al RHA de Yorkshire encontró que, por elegir uno de la larga letanía de ejemplos, se le habían adjudicado a esta autoridad un contrato a favor del «Yorkshire Water» para la incineración de residuos clínicos por valor de 7,2 millones de libras esterlinas de capital y dos millones al año de ingresos. No fue prestado de forma competitiva. Y no lo fue durante un período de quince años. La autoridad no obtuvo la aprobación de la Dirección de NHS. El problema era interorganizacional; la Dirección NHS central no podía dirigir (steer). La política del gobierno es una política de realización de propuestas de forma competitiva con carácter obligatorio. La política de la Unión Europea exige que los principales contratos del sector público esten abiertos a Estados miembros. Pero parecería que Yorkshire está exenta de ello ${ }^{8}$.

El centro dispone de mecanismos blandos (rubber levers); y, así, tirar de la palanca de la política central no significa necesariamente que algo ocurrirá en la periferia. Pese a varios intentos durante los años de fortalecer la capacidad central, el problema sigue estando ahí. La crítica llevada a cabo por el «White Paper» respecto de los departamentos centrales, la llamada a una aproximación corporativa, los cambios llevados a cabo para fortalecer la realización de políticas a nivel horizontal y el papel de la Oficina del Gabinete buscan todos ellos hacer frente al problema de la capacidad estratégica del centro. La coordinación es un tema central en el «White Paper», Modernizando al Gobierno.

La fragmentación erosiona la responsabilidad (accountability) porque la pura complejidad institucional no permite ver quién es responsable y por qué. Los organismos de objetivo determinado nominados se han multiplicado ocupando el lugar de los departamentos centrales y de los consejos locales electos para la prestación de algunos servicios. De nuevo, el RHA de Yorkshire ilustra de forma dramática esta cuestión; el catálogo de malas acciones salta a la vista. El Comité de Cuentas Públicas está «preocupado» con las ocho instancias de comportamiento «inaceptable», de lo que se dieron cuenta «con sorpresa» y «seria preocupación»; respecto de una de estas situaciones pronunciaron sentirse «espantados». También consideraron que la acción con carácter de remedio llevada a cabo había sido «profundamente insatisfactoria». El informe del Comité traza un dibujo de una autoridad que abrazó la cultura del momento, descuido su clásico papel de administrador, se salió con la suya respecto del blue murder durante varios años. Se ejerció control, pero más tarde, mucho más tarde. Nadie se vio obligado a dar explicaciones por el importante despilfarro de dinero públi- co. No se recuperó el dinero. Ni puede la RHA de Yorkshire ser descartada como una única y aberrante organización. Es un ejemplo, tanto de un gobierno privado en acción como del problema de hacer responder a los bolding networks.

Existe una confusión en el centro de la política, entre la capacidad de hacer responder y la responsabilidad. Resulta importante en dos sentidos. En primer lugar, la prestación de servicios con la capacidad de responder tal y como se contempló por tales innovaciones como los suplementos de la Carta de Derechos de los Ciudadanos, pero no viene a reemplazar la responsabilidad política porque el consumidor carece de poderes para hacer que la agencia del gobierno responda. Los ciudadanos se han convertido en los consumidores de servicios. La Carta de Derechos de los Ciudadanos es el equivalente del gobierno a la revista Wbich de la Asociación de Consumidores. Esta publicación pone a prueba tales bienes como lavadoras y televisores, y aconseja a los consumidores respecto de las mejores compras. ¿Resulta más efectiva la Carta de Derechos de los Ciudadanos que Which? Probablemente no. Éste es un debate importante respecto del apoderamiento (empowerment) de los ciudadanos para ejercitar un control democrático. En el gobierno británico fue un debate mudo, y ninguna de las políticas discutidas en este artículo ofrece un papel significante a los ciudadanos.

En segundo lugar, la agencificación agravó el «hueco» de responsabilidad porque el gobierno no introdujo nuevos arreglos para preservar la convención constitucional de responsabilidad ministerial. William WALDEGRAVE trató de justificar esta inacción estableciendo una distinción entre «responsabilidad, que puede ser delegada, responsabilidad, que sigue firmemente con el ministro». Desde este punto de vista, las agencias y otras reformas aclararon la responsabilidad, pero dejaron «al ministro como responsable propiamente por las políticas que él acuerda». Esta distinción gira en torno a definiciones claras tanto de política como de administración, y en torno a la distinción entre los papeles respectivos y las responsabilidades de los ministros, altos funcionarios civiles y jefes ejecutivos. Tal y como señaló la Tesorería y el Comité del Funcionariado Civil, no existen. Los arreglos que existen en la actualidad permiten que el ministro obtenga reconocimiento cuando la política vaya bien, pero permite a la vez poder culpar a los jefes ejecutivos cuando las cosas van mal -abundan los ejemplos-. No existe una línea divisoria clara entre las políticas y las operaciones, minando la responsabilidad ministerial frente al Parlamento al ayudar a los ministros a evitar cargar con las culpas ${ }^{9}$.

En resumen, el gobierno británico ha sufrido un significante descenso en la responsabilidad política. Éste es un problema principal, y no se limita únicamente al ámbito de las agencias. El Comité del Servicio Público quería una línea más directa de responsabilidad entre el Parlamento, los funcionarios y los jefes ejecutivos de las agencias ${ }^{10}$. El entonces gobierno con- 
servador rechazó estas propuestas. El Nuevo Laborismo mantiene en gran parte el silencio respecto de este asunto. $\mathrm{El}$ «White Paper» enfatiza «la capacidad de responder» frente a los ciudadanos como usuarios de servicios. Su consideración respecto de la responsabilidad va más allá de la remisión del Defensor del Pueblo parlamentario; fortaleciendo la auditoría; y la defensa de que los nombramientos ministeriales fortalecen la responsabilidad ( $\mathrm{Cm} 4310$, 1999: 7, 32, 38 y 60). La distinción de WALDEGRAVE entre responsabilidad administrativa por la prestación de servicios y responsabilidad ministerial frente al Parlamento está viva y bien, y el «White Paper» sólo hace frente a la primera.

El funcionariado civil ya no tiene el monopolio respecto de la consulta política. Los ministros insisten ahora en obtener consejo de diversas fuentes. Hasta el momento, sin embargo, no ha habido una abierta politización de los nombramientos del funcionariado del nivel superior. Los compromisos de Margaret Thatcher llevaron a Hugo Young a describir el servicio como «una satrapía profundamente thatcherizada», pero el grupo de trabajo del Instituto Real de las Administraciones Públicas llegó a la conclusión de que había habido «personalización, pero no politización» en los nombramientos superiores. Sin embargo, mientras que a lo mejor no hubo una politización de partido abierta de los altos funcionarios, hemos perdido el «escepticismo institucional». RICHARDS proporciona el resumen más convincente cuando argumenta que Thatcher «era despiadada en su determinación de tener funcionarios que fueran capaces de proporcionar respuestas a los problemas de implementación de las políticas de su gobierno»; los «administradores, y capaces de llevarlo a cabo» ${ }^{11}$.

Cuando el Nuevo Laborismo nombró 53 consejeros políticos, en comparación con los 38 que fueron nombrados bajo el gobierno anterior, vino a echar petróleo sobre las cenizas incandescentes del debate de la politización, tal y como hizo el intento, posteriormente abandonado, de nombrar a Jonathan Powell, el jefe de personal de Blair, como su principal secretario privado (un puesto normalmente ocupado por un funcionario). De nuevo, la búsqueda es la de funcionarios que puedan llevar a la práctica las políticas del gobierno. Algunos altos funcionarios civiles temen que, con la competencia abierta y los contratos, la era de los nombramientos políticos a los puestos de trabajo superiores ya esté con nosotros (información privada).

Un problema relacionado es el de los roles gerenciales. Los altos funcionarios civiles tienen tres funciones principales: el consejo político (a ministros), la administración (de sus departamentos) y la diplomacia (o gestión de las relaciones externas). Aconsejar al ministro no debe reducirse a dar consejo político. También tiene que ver con el apoyo y firefighting, y puede extenderse al área gris de los partidos políticos. Ahora los ministros se sirven de varias fuentes para obtener consejo político: consejeros políticos, think tanks, consultores. En general, el fun- cionariado civil ya no tiene un monopolio de asesoramiento. El funcionariado civil superior debe además asegurar que el departamento delivers, una frase que cubre tanto la organización del departamento como la gestión de los recursos humanos. Se centra en una gestión estratégica y una gestión operacional; la labor diaria del departamento cada vez se delega más y de forma más extensa. Por último, la diplomacia cubre todas las relaciones externas del departamento, es decir, sus relaciones con otros departamentos centrales, con otras agencias públicas (incluyendo las autoridades locales), con el Parlamento, los medios de comunicación y la Unión Europea. Tal y como ha observado Sir Douglas Wass, «la astucia y la diplomacia son un ingrediente esencial en el servicio público» ${ }^{12}$.

Los puntos de vista difieren hasta el punto de que el alto funcionariado civil ya ha adquirido algo más de una apariencia de nuevo gerencialismo. Los departamentos aún «están gestionados por gente que llegó allí por sus habilidades políticas más que por sus habilidades gestoras». RICHARDS señaló cómo mucho no ha cambiado, singularizando el reclutamiento fast stream; la responsabilidad ministerial; el secretario permanente como el único oficial responsable; la función de las políticas; procedimientos de nombramiento de los superiores; y la delegación del pago y de las condiciones de trabajo en las agencias. THEAKSTON concluye que las reformas han afectado al funcionariado civil superior de tres formas. En primer lugar, «no existe menos confianza en sí mismo del grupo». En segundo lugar, los funcionarios civiles superiores son rara vez los que inician las políticas; «son mucho menos activos a la hora de llevar adelante sus puntos de vista políticos». En tercer lugar, tienen un perfil público muy superior. Ya no son hombros del Estado camuflados. Pone de relieve su papel como «administrativos conservadores» que vigilan las instituciones, los procesos y el personal del gobierno y los valores y principios distintivos del servicio público. Sin embargo, hasta el momento, el cambio clave ha sido el hecho de que los funcionarios superiores se convirtieran en «gerentes de las políticas, más que creadores 0 iniciadores de las mismas» ${ }^{13}$.

Reformas recientes del sector público, especialmente reformas en la gestión corporativa y de las 3 e, supone un reto para la cultura de Whitehall. Muchos temen que se produzca una erosión de la ética del servicio público. Con la extensión del patronage y la existencia de un gobierno unipartidista durante dieciocho años, las preocupaciones acerca de los estándares de conducta crecieron. CHAPMAN y O'Toole alaban «las tradiciones de una burocracia apartidista, una situación actual que permitió al funcionariado acercarse a poner en práctica los valores de los guardianes de Platón y del tipo-ideal de burocracia de WEBER». Ellos defienden que «las virtudes del funcionariado británico tradicional, con su énfasis en la responsabilidad, y su aproximación casi vocacional a la motivación» en contra de «la búsqueda de moda de nuevas aproximaciones aparentemen- 
te nuevas a la gestión». Las nociones de responsabilidad frente al Parlamento a través del ministro y del deber público están en la base de su crítica. Argumentan que los funcionarios públicos deben aportar integridad, nunca poniendo sus intereses particulares antes del deber público, y deben ser objetivos e imparciales. Las reformas en la gestión atacaban, atacan al generalista y conducen a la «muerte del deber público». Las agencias fragmentan el funcionariado. Los funcionarios ya no se socializan en sus tradiciones compartidas. Los principios de la Carta de Derechos de los Ciudadanos sustituyen los rasgos distintivos del servicio público. Los valores tradicionales se perderán si no los protegemos. Los métodos gerenciales de tipo empresarial no son un sustituto adecuado ${ }^{14}$.

La gestión empresarial, la competencia abierta y los macho-ministers se suman a un reto frente a los rasgos distintivos de la ética del servicio público. De esta forma, creció el interés en un código ético. El gobierno conservador realizó un borrador de código (Cm 2748, 1995:5-6 y Anexo) y el Nuevo Laborismo propone darle fuerza de estatuto, aunque aún no se ha fijado el tiempo en el Parlamento para ello. Su Código Ministerial (Oficina del Gabinete, 1997: 21 para 56) establece que los ministros tienen el «deber de mantener la imparcialidad política del funcionariado» $\mathrm{y}$ «de asegurar que no se abuse de la influencia sobre los nombramientos de objetivos partidistas». El «White Paper» Modernizando el Gobierno también fija su compromiso con los servicios públicos y los funcionarios públicos $(\mathrm{Cm}$ 4310, 1997: ch. 6), aunque no «tolerará la mediocridad». Así, aún se mantiene la unidad del alto funcionariado.

\section{¿Se modernizará el gobierno?}

¿Conseguirán las propuestas del gobierno resolver alguno de estos problemas y permitirán alcanzar los objetivos fijados? Su política es más de lo mismo pero con una peculiaridad inesperada y ésta es que el gobierno conjunto. Cuatro cuestiones siguen sin resolverse: la coordinación, la mezcla de estructuras gubernamentales, la responsabilidad y los informes máximos de gestión.

La búsqueda de coordinación horizontal entre departamentos, y vertical entre las organizaciones del sector público se encuentra en el centro de las reformas del Nuevo Laborismo. Tal y como destacaron KaVANAGH y SELDON, hemos sido testigos de la centralización ministerial bajo el disfraz de innovación institucional y más recursos para la Unidad Número Diez y la Oficina del Gabinete; y una fuerte dirección política y de políticas, al igual que el Número Diez busca un control fuerte en la máquina gubernamental. El péndulo oscila de nuevo, a la vez que el centro promueve la coordinación y la vigilancia estratégica para combatir el departamentalismo de Whitehall. Tales «formas de agarrar el poder» son «una reacción ante la sentida debilidad, una frustración con la falta de habilidad para tirar de palancas efectivas» ${ }^{15}$.

Sin embargo, pese a las fuertes presiones para mayor coordinación y para que haya una coordinación proactiva a lo largo de toda Europa Occidental,

la coordinación de actividades básicas sigue siendo una práctica modesta en cuanto a su alcance: la mayor parte de la coordinación interna tiene lugar a niveles inferiores de la jerarquía estatal; es rara vez estratégica o incluso directiva, pero selectiva, orientada a cuestiones determinadas y reactiva; a la vez es negativa en el sentido de que se caracteriza por tolerar unidades fuertemente com. partamentalizadas que buscan la evitación mutua de estrategias para reducir las tensiones...

Todos los gobiernos han recurrido a una variedad de medidas para reducir el peso de la coordinación,... pero obteniendo sólo un éxito limitado y... muchas de estas medidas adoptadas han servido sólo para complicar e incluso aumentar los requisitos de la coordinación.

La coordinación es la piedra filosofal del gobierno moderno, siempre buscada, pero siempre más allá de nuestro alcance ${ }^{16}$.

Las zonas de acción muestran los límites de la coordinación. Hay una epidemia de zonas, hasta el punto de que la solución (hacia la fragmentación) se convierte en parte del problema (al añadir a los cuerpos que estén coordinados). Por ejemplo, John Denham, un ministro inferior en el Departamento de Salud, admite que «las zonas pueden a veces hacer que el gobierno para más bien más complicado que menos complicado a los ciudadanos» y existe el peligro de que «haya un exceso de iniciativas» porque las zonas no actúan conjuntamente ${ }^{17}$.

Las zonas muestran al gobierno adoptando una aproximación instrumental a la gestión de redes que presupone que el centro puede diseñar e imponer herramientas para proteger la integración entre redes y realizar los objetivos del gobierno central. Constituye un ejemplo de una tecnocracia consensual impuesta que no resolverá el problema de la coordinación. Las reformas tienen un empuje centralizador. Buscan coordinar departamentos y a las autoridades locales imponiendo un nuevo estilo de gestión a otras agencias. Así que «no quieren dirigir los servicios locales desde el centro», «pero el gobierno no teme actuar en aquellos ámbitos en los que se escapen de los estándares»; un ejemplo evidente de un código con órdenes operativas ( $\mathrm{Cm}$ 4310, 1999: 35, 37, 45, 53 y 55). Las zonas pertenecen al centro y las agendas locales son reconocidas en la medida en que faciliten la agenda central. Tal código no importa lo bien que esté disfrazado, corre el siempre presente riesgo de resultar recalcitrante en base a actores claves y a la pérdida de flexibilidad a la hora de hacer frente a problemas localizados. 
Una presión delicada aplicada insistentemente sigue constituyendo un código de órdenes operativas con un guante de seda. Cuando estamos sentados en lo más alto de la pirámide y no podemos ver la parte de abajo, los déficit en el control son una consecuencia no intencionada siempre presente. Las estructuras de redes se caracterizan por un estilo de negociación descentralizada que deja de lado el control en virtud de acuerdo. La gestión por medio de negociaciones supone ponerse de acuerdo respecto de los objetivos con los otros, no simplemente convencerles de que llevábamos razón todo el tiempo o recurrir a sanciones cuando no estamos de acuerdo.

Todas las estructuras de gobierno fallan. La burocracia y el red tape son una vieja letanía. También nos volvemos cada vez más conscientes de los límites de la marketización. Contracting-out permanece, pero la división comprador-proveedor desaparece y el PFI está acumulando problemas. El Tesoro puede evitar el gasto de capital, pero los «trusts» hospitalarios no disponen de recursos suficientes para pagar los costos del sector privado. Como ocurría con el gobierno anterior, los costes globales de estas políticas surgen lentamente. De forma similar, a límites a esas redes, son efectivos si siguen las siguientes condiciones:

- los actores necesitan información en la que puedan confiar e información más «gruesa»;

- la calidad no puede ser especificada o es difícil de definir y medir;

- los bienes son difíciles de tasar económicamente;

- la discreción profesional y la experiencia son valores claves;

- se requiere flexibilidad para hacer frente a demandas de servicios variadas y localizadas;

- se requiere coordinación entre sectores y entre agencias y producción;

- esta cooperación se enfrenta a culturas organizacionales dispares;

- los actores perciben el valor de las estrategias cooperativas;

- se necesitan relaciones a largo plazo para reducir la incertidumbre;

- el control y la evaluación incurren en altos costes administrativos y políticos; y

- la implementación implica regatear.

Del mismo modo, las redes, al igual que cualquier otro mecanismo de distribución, no son gratuitos. Éstos son:

- cerrados frente a los extraños a ellos y los que no son representativos;

- no son responsables por sus acciones;

- están diseñados para servir intereses privados, no el interés público (tanto al nivel local como nacional del gobierno);

- difíciles de dirigir (steer);
- ineficientes porque la cooperación ocasiona retrasos;

- inmovilizados por conflictos de intereses y

- difíciles de combinar con otras estructuras de gobierno.

También, la negociación de redes y la coordinación pueden confundirse con el contexto político en el que están inmersas. Rápidos niveles de cambio, conflictos sociales endémicos e intereses políticos a corto plazo, especialmente intereses de partido pueden, todos ellos, minar las negociaciones y la búsqueda de una forma de actuar acordada ${ }^{18}$.

Un efecto claro de la marketización es que mina la efectividad de las redes que extiende. El gobierno promovió la competencia y el contracting-out. El resultado fue «la corrosión... de los valores comunes y de los compromisos» y «crear una atmósfera de deconfianza». Las relaciones de mercado tuvieron «efectos corrosivos» sobre «las redes profesionales que dependen de la cooperación recíproca y de la interdependencia». En resumen, los contratos minan la confianza, la reciprocidad, la informalidad y la cooperación. Lo que importa es la mezcla de estructuras de gobierno; pero esa mezcla puede resultar ser como una mezcla de agua y aceite ${ }^{19}$.

Finalmente, antes de que este catálogo de cualificaciones y defectos sugiera que el gobierno conjunto y las redes no funcionan, es importante reiterar que todas las estructuras de gobierno fallan. Las redes tienen ventajas que cabe resaltar. En primer lugar, los mercados y las jerarquías fallan. Las redes funcionan en condiciones en que las anteriores no lo hacen. La lista de condiciones establecida arriba es la de condiciones bajo las cuales los mercados fallan; por ejemplo, donde es difícil especificar el precio del bien o del servicio. En segundo lugar, las redes juntan a los realizadores de políticas y a las agencias implementadoras, $\mathrm{y}$ al hacerlo, aumentan la experiencia e información disponible. En tercer lugar, las redes juntan también a muchos actores a negociar sobre una determinada política, aumentan la aceptabilidad de esa política y mejoran las probabilidades de que estén de acuerdo. En cuarto lugar, las redes incrementan los recursos disponibles para la realización de política acercando a los sectores público, privado y voluntario ${ }^{20}$.

El «White Paper» Modernizando al Gobierno no presta la menor atención a la responsabilidad política. El gobierno carece de propuestas para fortalecer la responsabilidad de los ministros frente al Parlamento. El énfasis recae sobre el control político central de prioridades y el control gerencial de la implementación. Modernizando el Gobierno propone una fijación tecnológica. Dejando el traspaso a un lado, la precaución política prevalece en la desconfianza del gobierno respecto de las autoridades locales, la decisión de dar carpetazo a las asambleas regionales en Inglaterra y las propuestas sobre «libertad» de información (Cm 3818, 1998; Cm 4355, 1999) ${ }^{21}$. Finalmente, las reformas del Nuevo Laborismo pueden empeorar el problema de la responsabilidad. HogwoOd, JUdGe y McVicar 
muestran que las agencias y los cuerpos con objetivo determinado tienen multiples electores, cada uno de ellos busca hacerles responsables, y no existe ningún sistema, sino sólo demandas dispersas y que se superponen. En las zonas, las organizaciones elegidas pueden hacer a sus oficiales y políticos responder - pero, cante quién son responsables esta serie de organizaciones?- Tal y como muestran Hood y sus colegas, el gobierno conjunto no supone una regulación conjunta, y nadie regula a los reguladores. En estas condiciones, la siguiente generación de RHAS de Yorkshire logrará medrar ${ }^{22}$.

En una conferencia, en mayo de 1999, sobre «El Funcionariado en el Nuevo Milenio», Sir Richard Wilson, jefe del «Home Civil Service», comentó:

«Mencioné con anterioridad el orgullo del que se ha servido el funcionariado de forma tradicional y que ha llevado a sus niveles superiores y su habilidad para aconsejar a los Ministros sobre políticas. Pero ahora estamos empezando a cuestionar entre nosotros lo buenos que éramos de hecho con estas habilidades. ¿Hablábamos acaso de diseñar políticas que podían ser gestionadas de forma efectiva para proporcionar los resultados que el gobierno de ese momento quería? ¿O estábamos más preocupados en diseñar políticas que el ministro podía hacer pasar a través de sus colegas de gabinete y del Parlamento y presentar con éxito ante la prensa? ¿Y que a menudo hemos en la práctica vuelto atrás después y hemos evaluado el éxito con el que las políticas han proporcionado lo que se les pedía que hicieran en ese momento cuando eran impulsadas, más que simplemente llevadas hacia adelante para diseñar la siguiente política que ayuda al ministro a superar los momentos difíciles?»

Puede haber preguntas. El cambio no es tan obvio. En el año 2000, un secretario permanente es blanco tiene más de cincuenta años, con un título de Oxbridge en humanidades, que ha trabajado como funcionario de veinticinco a treinta años, sirviendo en un ministerio tan central como funcional, y que se jubilará a los sesenta después de cinco a diez años en un puesto máximo. Y, por supuesto, será varón; entre 1970 y 1998, hubo sólo cinco mujeres que fueran secretarios permanentes, y dos de ellas todavía tienen el puesto. Sus tareas siguen siendo una mezcla de aconsejar en cuestiones políticas, gestión y diplomacia, aunque el balance tenga sus cambios. Los años ochenta y noventa fueron testigos de un cambio en el énfasis que pasó de estar en el consejo político a estar en la gestión, y un gobierno conjunto contempla un cambio aún mayor hacia la diplomacia. El reclutamiento de funcionarios se ha vuelto cada vez más diverso. Su escenario es menos socialmente exclusivo. Los cambios recientes se abrirán camino hacia lo más alto. En 1998, alrededor de la mitad de los reclutados para puestos superiores eran de Oxbridge y dos tercios tenían títulos en arte y literatura. Los patrones y los procedimientos de reclutamiento comen- zaron a cambiar en los años noventa. Los departamentos y las agencias sólo necesitan tomar en consideración la competencia abierta, y no todas las vacantes están abiertas para candidatos externos. Alrededor del $30 \%$ de todas las vacantes en puestos superiores están abiertas ahora a candidatos externos, aunque el informe de Sir Richard Wilson contempla otro empuje para hacer el servicio más diverso (Oficina del Gabinete, 1999c). El cliché apto es el de «evolución, no revolución»; el cambio afecta «a todos, menos a nosotros».

El «White Paper» Modernizando el Gobierno no llevará a un aumento dramático del grado de cambio. No hubo propuestas para evaluar el consejo político y el trabajo de los funcionarios civiles superiores. Sin embargo, hay una pista leve de las cosas que están en camino ( $\mathrm{Cm}$ 4310, 1999; y la Oficina del Gabinete, 1999b y c). De esta forma, el informe llevado a cabo por el Equipo de Realización de Políticas Estratégicas trata de fijar estándares para mejorar la realización de políticas e incluye peer review (Oficina del Gabinete, 1999b: para 1.4 y 11,4-11,6). Sin embargo, este informe se centra en el análisis político que tiende a realizarse a grados inferiores al de secretario permanente. En este mismo sentido, Sir Richard Wilson contempla «informes rigurosos que comprenden a todos los gestores». Una cuestión es clara, después de veinte años de reforma continuada, para diciembre de 1999 todavía no existían procedimientos sistemáticos para evaluar el trabajo de los administradores superiores, ya fuera en política, gestión o diplomacia.

Puede llevarse a cabo. Weller y Stevens describen los «Informes Australianos de la Gestión Pública» (de la calidad del consejo político). Los tópicos incluyen el papel del departamento del Primer Ministro y del Gabinete en el comité interdepartamental en previsión al cuidado de las personas ancianas; la evaluación de los papeles de la «Forward Estimates Strategy» para el presupuesto del año 93-94; y el informe de los procesos de reforma del estado del Commonwealth. Estos informes consultaron documentos oficiales y entrevistaron a actores claves. Debido a que el PMRs evaluaba de forma central y no en línea a los departamentos, estaban más preocupados con inputs y con el proceso, que con los resultados, y fue difícil identificar el valor añadido por el centro. Sin embargo, PMRs explicó lo que había ocurrido e identificó las lecciones que fueron utilizadas para adiestrar a los funcionarios superiores. Tal y como concluyen los autores: «Ninguno de los descubrimientos ha sido sorprendente... reflejan y desarrollan la inteligencia... pero no proporcionan una fórmula para poder examinar los procesos de forma estructurada que resulta extraña al ritmo frenético del gobierno central. La resistencia de la élite administrativa británica a aplicarse a sí misma los sistemas de auditoría y de evaluación que ha impuesto a los demás puede provocar una demanda de nombramientos políticos» ${ }^{23}$. 


\section{Conclusión: la necesidad de articularse}

El Nuevo Laborismo rechaza el modelo de órdenes burocrático del Viejo Laborismo con su énfasis en la jerarquía, la autoridad y las reglas. A primera vista, acepta la política del gobierno conservador de marketizar los servicios públicos; pero acepta pragmáticamente tales reformas como la Carta de Derechos de los Ciudadanos a la vez que rechaza otras. De forma distintiva, defiende «un gobierno conjunto», o la prestación de servicios por medio de redes de organizaciones que dirigen (steer), donde lo normal no es la autoridad (burocracia) o la competencia en los precios (mercados), sino la confianza. En el lenguaje de las clase habladoras, es la «tercera vía» en la acción. Ejemplifica el cambio del Estado proveedor del Viejo
Laborismo y el Estado mínimo del Thatcherismo al Estado capaz. Los funcionarios siguen combinando el consejo político, la gestión y la diplomacia, pero la llegada del gobierno conjunto $\mathrm{da}$ indicios de un posterior cambio de la gestión a la diplomacia.

Hay mucho que agradecer en el programa modernizador del Nuevo Laborismo, central, local y de gobierno traspasado. Pero el gobierno carece de la confianza que trata de inspirar. Teme la independencia que concede. Así que el «White Paper» sobre Modernizar el Gobierno reconoce la necesidad de gestionar las redes, pero no consigue reconocer los límites, tanto de la intervención central a medida que intenta equilibrar la independencia con el control central y con las redes como estructura de gobierno que puede fallar. La lección más difícil de aprender para el gobierno británico central es la de «mano fuera» o «no tocar».

\section{Traducción: Cristina AlBA MuÑoz.}

' Realizo una visión general del periodo inicial en R. A. W. RHODEs, Understanding Governance, Buckingham: Open University Press, 1997, chs. 5-7. Ver también A. Gray y B. Jenkins, «New Labour, New Government? Change and Continuity in Public Administration and Government 1997», Parliamentary Affairs, 1998, pp. 111-130; P. Hennesssy, «The Blair Style of Government», Government and Opposition, 1998, pp. 3-20; K. ThEakston, «New Labour, New Whitehall?», Public Policy and Administration, 1998, pp. 13-33. De lo que peca y por lo que es perseguido este tipo de análisis es de centrarse en intenciones elitistas y no en resultados, tal y como afectan a varios de los apostantes. También, tal y como $\mathrm{C}$. PoLLITT argumenta persuasivamente en «Justification by Works or by Faith? Evaluating the New Public Management», Evaluation, 1995, pp. 133-154, de hecho, las reformas son un acto de fe rara vez evaluado y a menudo las reformas son, a su vez, reformadas, antes de que sus resultados se vean de forma clara. Cuando ha sido posible, he tratado de evaluar las reformas, en contra de la prueba existente, pero, inevitablemente, en mi análisis hay un elemento de especulación.

Entre abril de 1994 y junio de 1999 fui Director del Consejo de Investigación Económica y Social (ESRC), Programa Whitehall sobre la naturaleza cambiante del gobierno británico central. Este artículo se sirve de mi trabajo en ese Programa. Para un informe completo relativo a esta investigación, ver R. A. W. RHODEs, Transforming British Government, vol. 1, Changing Institutions; vol. 2, Changing Roles and Relationships, London: Macmillan, 2000.

${ }^{2}$ Para evitar numerosas notas de pie de página, en el texto I, señalo cuáles son las publicaciones oficiales relevantes, bien por medio de sus números de control, y su fecha de publicación, o su origen departamental y su fecha de publicación. Las fuentes principales son: $\mathrm{Cm} 2627$ (1994), The Civil Service: Continuity and Change, London: HMSO; Cm 2748 (1995), The Civil Service: Taking Forward Continuity and Change, London: HMSO; Cm 2850 (1995), Standards in Public Life: The First Report of the Committee on Standards in Public Life (Nolan), London: HMSO; Cm 3658 (1997), Scotland's Parliament, London: The Stationery Office; Cm 3889 (1998), Next Steps Report 1997, London: The Stationery Office; Cm 4011 (1998), Modern Public Services for Britain: Investing in Reform. Comprebensive Spending Review: New Public Spending Plans 1999-2002, London: The Stationery Office; Cm 4014 (1998), Modern Local Government: In Touch with The People, London: The Stationery Office; Cm 4181 (1998), Public Services for the Future: Modernization, Reform, Accountability, London: The Stationery Office; $\mathrm{Cm} 4310$ (1999), Modernizing Government, London: The Stationery Office; Cm 4355 (1999), Freedom of Information: Consultation on Draft Legislation, London: The
Stationery Office; Cm 4444 (1999), Memorandum of Understanding and Supplementary Agreement between the United Kingdom Government, Scottish Ministers and the Cabinet of National Assembly for Wales, London: The Stationery Office; Cabinet Office (1994), Next Steps: Moving On (The Trosa Report), London: Cabinet Office; Cabinet Office (1997), Ministerial Code: A Code of Conduct and Guidance on Procedures for Ministers, London: Cabinet Office; Cabinet Office (1998), Service First: The New Charter Programme, London: Cabinet Office, junio; Cabinet Office (1999a), Modernizing Government Action Plan, London: Cabinet Office, julio; Cabinet Office (1999b), Professional Policy Making for the Twentieth Century: Report by the Strategic Policy Making Team, London: Cabinet Office, septiembre; and the Cabinet Office (1999c), Report to the Prime Minister from Sir Richard Wilson, Head of the Home Civil Service, Civil Service Reform, London: Cabinet Office, 15 diciembre.

${ }^{3}$ Ver M. POWER, The Audit Explosion, London: Demos, 1994, pp. 8-9.

${ }^{4}$ C. Hood, O. James, G. Jones, C. Scott y T. Travers, «Regulation Inside Government: Where New Public Management Meets the Audit Explosion», Public Money and Management, 1998, pp. 61-68; C. Hood, O. JAMES y C. SCOTT, «Regulation in Government: Has It Increased, Is It Increasing, Should It Be Diminished?», Public Administration, 2000, a punto de publicarse. Ver también Tony Blair, «Modernising Public Services», discurso ante los Charter Mark Awards, Central Hall, Westminster, 26 de enero de 1999.

5 P. DaY y R. KLEIN, Steering But Not Rowing? The Transfomation of the Department of Health: A Case Study, Bristol: Policy Press, 1997.

${ }^{6}$ Hennessy, The Blair Style of Government, p. 15.

${ }^{7}$ W. I. JENKINS, «Reshaping the Management of Government: The Next Steps Initiative in the United Kingdom», en F. L. SEIDLE (ed.), Retbinking Government: Reform or Revolution?, Quebec: Institute for Research on Public Policy, 1993, p. 94; y Sir Robin Butler, «The Evolution of the Civil Service», Public Administration, 1993, p. 404.

${ }^{8}$ Committee of Public Accounts, The Former Yorkshire Regional Health Authority: The Inquiry Commissioned by the NHS Chief Executive, London: The Stationery Office, 1997.

${ }^{9}$ Ver W. Waldegrave, Public Service and the Future: Reforming Britain's Bureaucracies, London: Conservative Political Centre, 1993, p. 20; Treasury and Civil Service Committee, Role of the Civil Service, London: HMSO, 1994, p. 132; W. Plowden, Ministers and Mandarins, London: Institute for Public Policy 
Research, 1994, p. 127; J. STEWART, «Defending Public Accountability», Demos Newsletter, noviembre 1993, pp. 5-10.

${ }^{10}$ Public Service Committee, Ministerial Accountability and Responsibility, HC 313, Session 1995-1996, London: The Stationery Office, 1996, chs. 4 y 5.

$"$ Tomado, literalmente, de P. HenNEssy, Whiteball, London: Secker and Warburg, 1989, p. 631. Ver también, RIPA, Top Jobs in Whiteball: Appointments and Promotions in the Senior Civil Service, London: RIPA, 1987, p. 43; PLOWDEN, Ministers and Mandarins, p. 104; y D. RicHARDs, The Civil Service under the Conservatives 1979-1997, Brighton: Sussex Academic Press, 1997, pp. 123-124.

12 Tomado literalmente, de Hennessy, The Blair Style of Government, p. 150.

${ }^{13}$ S. WATSON, «Is Sir Humphrey Dead? The Changing Culture of the Civil Service», Working Paper, No. 103, Bristol: School for Advanced Urban Studies, 1992 p. 27; K. THEAKSTON, Leadership in Whitehall, London: Macmillan, 1999, pp. 257-259; P. BARBERIS, The Elite of the Elite, Aldershot: Dartmouth, 1996 p. 42; y S. RICHARDS, «Memorandum Submitted by Professor Sue Richards, Director of the Public Management Foundation», en Treasury and Civil Service Committee, Role of the Civil Service, vol. II, Minutes of Evidence and Appendices, London: HMSO, 6th Report. HC 390-II, Session 1992-3, pp. 277-280.

14 R. Chapman y B. J. O'Toole, «The Role of the Civil Service: A Traditional View in A Period of Change», Public Policy and Administration, 1995, pp. 4, 5-7.

15 D. Kavanagh y A. Seldon, «The Power Behind the Prime Minister: The Hidden Influence of No.10», en RHODES (ed.), Transforming British Government, vol. 2, Cbanging Roles and Relationahips.

${ }^{16}$ J. E. S. Hayward y V. Wright, «Governing from the Center: Policy Coordination in Six European Core Executives», en RHODEs (ed.), Transforming British Government, vol. 2, Changing Roles and Relationships.

${ }^{17} \mathrm{~J}$. Denham, discurso dirigido a CIPFA/Public Management and Policy Association Conference sobre una «Epidemia de Zonas: Enfermedad o Cura», International Conference Centre, Birmingham, junio 1999. D. WALKER, «Methods in the Zones Mosaic?, Public Finance, 11-17 de juno de 1999, pp. 24-6, también identifica algunas otras debilidades en las zonas. Por ejemplo, recortan las competencias de las autoridades, divergen fondos de los programas principales, y coste, tiempo y dinero; $y$, de forma aún más importante las causas estructurales de problema como la pobreza son regionales, nacionales e internacionales, y no locales. Los Programas de Desarrollo de la Comunidad de los años setenta nos enseñaron esta lección, pero tales críticas parecen caer dentro de la categoría del «conocimiento que resulta un inconveniente», y que es mejor ignorar.

${ }^{18}$ En las condiciones bajo las cuales las redes prosperan, y para citas relevantes, ver R. A. W. RHODES, «It's the Mix that Matters: From Marketization to Diplomacy», Australian Journal of Public Administration, 1997, pp. 40-53; y W. Powell, «Neither Market nor Hierarchy: Network Forms of Organization», en G. Tномр. son y otros eds., Markets, Hierarchies and Networks: The Coordination of Social Life, London: Sage, 1991.

${ }^{19}$ R. FlynN et al., Markets and Networks: Contracting in Community Services, Buckingham: Open University Press, pp. 115, 136-137.

${ }^{20}$ Para una discusión acerca de formas prácticas para ayudar a estos sectores a trabajar conjuntamente, ver Perri 6, D. Leat, K. Seltzer y G. Stoker, Governing in the Round:Strategies for Holistic Governance, London: Demos, 1999; G. STOKER, «Will Government Ever Get Joined Up?», paper presentado al ESRC Whitehall Programme Public Service Seminar, British Academy, London, 17 de septiembre de 1999; y D. WILKInson y E. APPelBeE, Implementing Holistic Government, London: Demos, 1999.

${ }^{21}$ No resulta probable que la política del Nuevo Laborismo respecto de la libertad de información vaya en modo alguno a alterar este análisis. El «White Paper» del gobierno $(\mathrm{Cm} 3818,1997)$ trató de hacer más responsable al gobierno frente al pueblo. La intención era hacer que toda la información estuviera disponible salvo que fuera dañina para la seguridad nacional, la discusión política interna, la aplicación de las leyes, la privacidad personal, los «trade secrets», la seguridad pública y sus referencias, y otras cuestiones dadas en confianza. El «White Paper» sorprendió a los comentaristas, muchos de los cuales lo contemplaron como una gran mejora. Este optimismo, sin embargo, no sobrevivió la transferencia de responsabilidad por parte de la legislación de la Oficina del Gabinete, «Home Office». La Constitución del derecho a la libertad de información (1999) «parece contener más excepciones que el White Paper o el Código de Práctica», incluyendo una exención general «que sea necesario para permitir que se tome en cuenta el interés público en circunstancias que, aunque no están exentas dentro de la ley, sin embargo, no estaría en favor del interés público que la información en cuestión se revelará» ( $\mathrm{Cm} 4355,1999: 8-11)$.

${ }^{22}$ B. W. HogwoOD, D. JUdGe y M. McVICAR, «Agencies and Accountability», en RHODEs (ed.), Transforming British Govermment, vol. 1, Changing Institutions.

${ }^{23}$ M. WALLer, «Evaluating Policy Advice», Australian Joumal of the Public Administration, 1992, pp. 440-446; P. Weller y B. Stevens, «Evaluating Policy Advice: The Australian Experience», Public Administration, 1998, pp. 579-589. 